\title{
Chromatin Regulation by HP1 $\gamma$ Contributes to Survival of 5-Azacytidine-Resistant Cells
}

\author{
Satoshi Imanishi ${ }^{1 *}$, Tomohiro Umezu ${ }^{1}$, Chiaki Kobayashi ${ }^{1}$, Tomohiko Ohta ${ }^{2}$, \\ Kazuma Ohyashiki ${ }^{3}$ and Junko H. Ohyashiki ${ }^{1}$
}

1 Institute of Medical Science, Tokyo Medical University, Tokyo, Japan, ${ }^{2}$ Department of Translational Oncology, St. Marianna University Graduate School of Medicine, Kawasaki, Japan, ${ }^{3}$ Department of Hematology, Tokyo Medical University, Tokyo, Japan

Recent investigations of the treatment for hematologic neoplasms have focused on targeting epigenetic regulators. The DNA methyltransferase inhibitor 5-azacytidine (AZA) has produced good results in the treatment of patients with myelodysplastic syndromes. The mechanism underlying its pharmacological activity involves many cellular processes including histone modifications, but chromatin regulation in AZAresistant cells is still largely unknown. Therefore, we compared human leukemia cells

\section{OPEN ACCESS}

Edited by:

Anna Rita Migliaccio,

Icahn School of Medicine at Mount

Sinai, United States

Reviewed by:

Chiara Ambrogio,

Dana-Farber Cancer Institute,

United States

Shiv K. Gupta,

Mayo Clinic, United States

*Correspondence:

Satoshi Imanish

s-ima@tokyo-med.ac.jp

Specialty section:

This article was submitted to

Cancer Molecular Targets

and Therapeutics,

a section of the journa

Frontiers in Pharmacology

Received: 30 June 2018

Accepted: 26 September 2018

Published: 16 October 2018

Citation:

Imanishi S, Umezu T,

Kobayashi C, Ohta T, Ohyashiki K and Ohyashiki JH (2018) Chromatin Regulation by HP1 $\gamma$ Contributes

to Survival of 5-Azacytidine-Resistant

Cells. Front. Pharmacol. 9:1166.

doi: 10.3389/fphar.2018.01166 with AZA resistance and their AZA-sensitive counterparts with regard to the response of histone modifications and their readers to AZA treatment to identify novel molecular target(s) in hematologic neoplasms with AZA resistance. We observed an a decrease of HP1 $\gamma$, a methylated lysine 9 of histone $\mathrm{H} 3$-specific reader protein, in AZA-sensitive cells after treatment, whereas AZA treatment did not affect HP1 family proteins in AZAresistant cells. The expression of shRNA targeting HP1 $\gamma$ reduced viability and induced apoptosis specifically in AZA-resistant cells, which accompanied with down-regulation of ATM/BRCA1 signaling, indicating that chromatin regulation by HP1 $\gamma$ plays a key role in the survival of AZA-resistant cells. In addition, the amount of HP1 $\gamma$ protein in AZAsensitive and AZA-resistant cells was decreased after treatment with the bromodomain inhibitor I-BET151 at a dose that inhibited the growth of AZA-resistant cells more strongly than that of AZA-sensitive cells. Our findings demonstrate that treatment with AZA, which affects an epigenetic reader protein and targets HP1 $\gamma$, or a bromodomain inhibitor is a novel strategy that can be used to treat patients with hematopoietic neoplasms with AZA resistance.

Keywords: drug resistance, azacytidine, histone modification, heterochromatin protein 1, bromodomain inhibitor, leukemia

\section{INTRODUCTION}

In recent years, the treatment for hematologic neoplasms has focused on targeting epigenetic regulators, such as DNA methyltransferases, histone deacetylases, and bromodomain proteins, as well as tyrosine kinases and proteasomes. The DNA methyltransferase inhibitor AZA has produced good results in the treatment of patients with myelodysplastic syndromes. Because of its DNA

Abbreviations: AZA, 5-azacytidine; HP1, heterochromatin protein 1; shHP1 $\gamma$, shRNA targeting HP1 $\gamma$. 
demethylation activity, most studies of the mechanism underlying the pharmacological activity of AZA have focused on the reactivation of tumor suppressor genes via demethylation of DNA in the promoter region (Lund et al., 2014; Tsujioka et al., 2015; Achille et al., 2016). However, recent studies have demonstrated the involvement of aberrant RNA metabolism (Aimiuwu et al., 2012), disruption of ribosome biogenesis (Moss, 2011), DNA damage response (Stresemann and Lyko, 2008), and histone modifications (Komashko and Farnham, 2010; Grövdal et al., 2014) in AZA activity, suggesting that the mechanism underlying its pharmacological activity is more complex than initially considered.

In parallel with research on the pharmacological activity of AZA, other studies have revealed the molecular mechanisms involved in AZA resistance. The importance of down-regulation of pyrimidine salvage in AZA resistance, which results in reduced phosphorylation of AZA, is well established (Saunthararajah, 2013; Imanishi et al., 2014; Valencia et al., 2014). The correlation of aberrantly high expression of ribosomal RNA with AZA resistance and the involvement of constitutive activation of the DNA damage response and DNA hypomethylation in AZA resistance have also been reported (Imanishi et al., 2014; Monika Belickova et al., 2016). However, chromatin regulation in AZAresistant cells is still largely unknown.

In this study, we investigated the dynamics of histone modifications and their reader proteins in AZA-resistant cells to examine the possibility that they may be the novel targets in AZA-resistant hematologic neoplasms.

\section{MATERIALS AND METHODS}

\section{Cells and Reagents}

U937 and HL-60 cells were purchased from ATCC (Manassas, VA, United States). AZA-resistant cell lines (R-U937 and R-HL60) were originally created in our laboratory from U937 and HL-60 cells, respectively (Imanishi et al., 2014).

5-Azacytidine was purchased from Wako Pure Chemical Industries (Osaka, Japan) and I-BET151 from Selleck Chemicals (Houston, TX, United States). The antibodies specific for HP1 $1 \alpha, \mathrm{HP} 1 \beta, \mathrm{HP} 1 \gamma$, and $\beta$-actin were purchased from Abcam (Cambridge, United Kingdom). The antibodies specific for BRCA1 phosphorylated at Ser 1423 (p-BRCA1), total BRCA1 and total ATM were from Santa Cruz Biotechnology (Dallas, TX, United States) and anti ATM phosphorylated at Ser 1981 (p-ATM) antibody was from R\&D Systems (Minneapolis, MN, United States). Anti PARP1 antibody was from Cell Signaling Technologies (Danvers, MA, United States). The secondary antibodies, namely horseradish peroxidase-labeled anti-mouse IgG antibody and horseradish peroxidase-labeled anti-rabbit IgG antibody, were purchased from GE Healthcare (Buckinghamshire, United Kingdom).

\section{Cell Culture and Chemical Reagent Treatment}

U937, HL-60, R-U937, and R-HL-60 cells were incubated in RPMI1640 medium (Life Technologies Inc., Carlsbad, CA,
United States) including 10\% inactivated fetal bovine serum and $1 \%$ penicillin/streptomycin (Life Technologies Inc., Carlsbad, CA, United States). For treatment with reagents, cells were collected by centrifugation and suspended at $1 \times 10^{5}$ cells $/ \mathrm{ml}$ in fresh medium with the reagents or $0.01 \%$ DMSO as the vehicle. Cell viability was measured using the Cell Counting Kit8 (Dojindo, Kumamoto, Japan) as previously described (Umezu et al., 2013).

\section{Western Blotting}

Western blotting of HP $1 \alpha$, HP $1 \beta, H P 1 \gamma$, p-BRCA1, total BRCA1, p-ATM, total ATM, PARP1 and $\beta$-actin was performed using whole cell lysate as previously described (Umezu et al., 2013). Briefly, the membranes were probed with antibodies directed against HP1 $\alpha$ (1:500), HP1 $\beta$ (1:500), HP1 $\gamma$ (1:500), p-BRCA1 (1:500), total BRCA1 (1:500), p-ATM (1:200), total ATM (1:200), PARP1 (1:500), or $\beta$-actin $(1: 1000)$ and then treated with the appropriate secondary antibodies.

\section{Establishment of Doxycycline-Inducible shHP1 $\gamma$ Transfected Cells}

The plasmid used in this study, CS-RfA-shHP1 $\gamma \# 1$-ETBsd, was prepared as previously reported (Wu et al., 2015). This plasmid expresses shHP1 $\gamma$ by treatment with doxycycline. Lentivirus containing CS-RfA-shHP1 $\gamma \# 1-E T B s d$ or CS-RfA-ETBsd as a negative control was prepared using the Single Shot Lenti$\mathrm{X}$ Packaging Kit (Takara Bio, Ohtsu, Japan) and Lenti-X 293 cells (Takara Bio). U937, HL-60, R-U937, and R-HL-60 cells were suspended in the medium containing lentivirus at $1 \times 10^{5}$ cells $/ \mathrm{ml}$, and medium containing fresh lentivirus was added at 24 and $48 \mathrm{~h}$. After incubation for $72 \mathrm{~h}$, the cells were incubated in fresh medium containing $10 \mu \mathrm{g} / \mathrm{ml}$ blasticidin for 7 days. The surviving cells were maintained in medium containing $10 \mu \mathrm{g} / \mathrm{ml}$ blasticidin. To induce the expression of $\operatorname{shHP} 1 \gamma$, the cells were treated with $1 \mu \mathrm{g} / \mathrm{ml}$ doxycycline.

\section{Quantitative RT-PCR}

Quantitative RT-PCR was performed as previously described (Ohyashiki et al., 2005). Total RNA was purified from AZA treated cells, shHP1 $\gamma$ transfected cells or negative control cells incubated in the presence of $1 \mu \mathrm{g} / \mathrm{ml}$ doxycycline for 96 h. TaqMan gene expression assays (Life Technologies Inc., Carlsbad, CA, United States) were used for CBX5 coding HP1 $\alpha$ (Hs01127577_m1), CBX1 coding HP1 $\beta$ (Hs01080635_g1), and CBX3 coding HP1 $\gamma$ (Hs04234989_g1). TaqMan PreDeveloped Assay Reagent (Life Technologies Inc., Carlsbad, CA, United States) was used for $A C T B$. The expression level of $C B X 1,3$ and 5 relative to the $A C T B$ expression level was determined by the $\triangle \mathrm{CT}$ method.

\section{Flow Cytometric Analysis of Apoptosis}

The FITC Annexin V Apoptosis Detection Kit I (BD Biosciences, San Jose, CA, United States) was used. Cell lines treated with doxycycline for 4 days were suspended in binding buffer and incubated with FITC-labeled annexin $\mathrm{V}$ and propidium iodide in the dark. 
Flow cytometric measurements were performed on a $\mathrm{BD}$ Accuri C6 Flow Cytometer (BD Biosciences, San Jose, CA, United States). A 488-nm blue laser was used for excitation, and signals were detected using the FL1 channel $(533 \mathrm{~nm}$ ) for FITC and the FL2 channel (585 nm) for propidium iodide. The signals of 30,000 events were obtained. Analyses of the obtained data were performed by using C6 software version 1.0 (BD Biosciences, San Jose, CA, United States).

\section{Statistical Analyses}

For statistical analyses, two-way ANOVA followed by the $t$-test were performed by using GraphPad PRISM 6 software (GraphPad Software Inc., La Jolla, CA, United States); $p<0.05$ was considered significant. Data are shown as mean $\pm \mathrm{SD}$ in the figures, and they represent the results obtained from three independent experiments.

\section{RESULTS}

\section{AZA Treatment Affected HP1 Family Proteins in AZA-Sensitive Cells but Not in AZA-Resistant Cells}

To investigate the chromatin regulation in AZA-resistant cells, we focused on HP1 proteins, the specific readers of di- or trimethylated lysine 9 of histone $\mathrm{H} 3$ (H3K9), because previous studies showed that AZA treatment affected on the modifications of H3K9 (Grövdal et al., 2014; Tobiasson et al., 2017). The amounts of HP1 $\alpha$ protein in U937, R-U937, HL-60, and R-HL60 were not affected by AZA treatment (Figure 1A). In HL-60 cells treated with $5 \mu \mathrm{M}$ AZA for $72 \mathrm{~h}$, a four-fold decrease of HP1 $\beta$ was detected, whereas AZA treatment had no clear effect on the amount of HP1 $\beta$ in U937, R-U937, and R-HL-60 cells. Although a remarkable decrease in the amount of HP1 $\gamma$ was found in both U937 cells and HL-60 cells after AZA treatment, no such changes were detected in R-U937 and R-HL-60 cells. In HP1 $\alpha$ mRNA expression, we did not detect any change in U937 cells, HL-60 cells, R-U937, and R-HL-60 cells after $5 \mu \mathrm{M}$ AZA treatment for $72 \mathrm{~h}$ (Figure 1B). HP1 $\beta$ mRNA expression in U937 cells, R-U937 and R-HL-60 cells was not affected by $5 \mu \mathrm{M}$ AZA treatment for $72 \mathrm{~h}$, while that in HL-60 cells was significantly reduced after $5 \mu \mathrm{M}$ AZA treatment for $72 \mathrm{~h}$ (Figure 1C). The mRNA expression of HP1 $\gamma$ was decreased in U937 cells and HL60 cells, but not in R-U937 cells and R-HL-60 cells, after treatment with $5 \mu \mathrm{M}$ AZA for $72 \mathrm{~h}$ indicating that AZA treatment repressed the transcription of HP1 $\gamma$ mRNA (Figure 1D). These results indicated that $A Z A$ treatment disrupted chromatin regulation via the methylated $\mathrm{H} 3 \mathrm{~K} 9 / \mathrm{HP} 1 \gamma$ axis in AZA-sensitive cells but not in AZA-resistant cells.

\section{Targeting HP1 $\gamma$ Induced Apoptosis Specifically in AZA-Resistant Cells}

To clarify the role of HP1 $\gamma$ in AZA-resistant cells, we established cells expressing shRNA targeting HP1 $\gamma$ mRNA in a Tet-On system-dependent manner. As shown in Figure 2A, HP1 $\gamma$ mRNA significantly decreased after incubation for $72 \mathrm{~h}$ in the presence of $1 \mu \mathrm{g} / \mathrm{ml}$ doxycycline, demonstrating that HP1 $\gamma$ expression was successfully knocked down. The incubation with doxycycline for $96 \mathrm{~h}$ did not affect the viability of shHP1 $\gamma$-infected U937 cells and shHP1 $\gamma$-infected HL-60 cells or in negative control cells, whereas the growth of shHP1 $\gamma$-infected R-U937 cells and shHP1 $\gamma$-infected R-HL-60 cells was inhibited by doxycycline treatment (Figure 2B). In the analyses of annexin $\mathrm{V} /$ propidium iodide (PI) double staining, treatment with doxycycline for $96 \mathrm{~h}$ increased the annexin V-positive/PInegative and annexin $\mathrm{V}$-positive/PI-positive populations in shHP1 $\gamma$-infected R-U937 cells and shHP1 $\gamma$-infected R-HL-60 cells (Figure 3A, lower panels) but not in shHP1 $\gamma$-infected U937 cells and shHP1 $\gamma$-infected HL-60 cells (Figure 3A, upper panels). The increase of cleavage of PARP1 was detected in shHP1 $\gamma$-infected R-U937 cells and shHP1 $\gamma$-infected R-HL-60 cells treated with doxycycline, while the presence of doxycycline did not affect on the PARP1 cleavage in shHP1 $\gamma$-infected U937 cells and shHP1 $\gamma$-infected HL-60 cells (Figure 3B). These results suggested that targeting HP1 $\gamma$ was cytotoxic specifically in AZAresistant cells.

\section{Targeting HP1 $\gamma$ Interfered ATM/BRCA1 Signaling in AZA-Resistant Cells}

To understand the role of HP1 $\gamma$ in AZA-resistant cells, we investigated the effect of HP1 $\gamma$ targeting on phosphorylation of ATM and BRCA1 proteins because these proteins are constitutively activated in AZA-resistant cells as we previously reported (Imanishi et al., 2014). As shown in Figure 4, p-ATM, but not p-BRCA1, was detected in shHP1 $\gamma$-infected U937 cells and shHP1 $\gamma$-infected HL-60 cells after incubation with doxycycline for $72 \mathrm{~h}$ indicating HP1 $\gamma$ was need to phosphorylate BRCA1 by active ATM in AZA-sensitive cells. P-ATM and p-BRCA1 were detected in shHP1 $\gamma$-infected R-U937 cells and shHP1 $\gamma$-infected R-HL-60 cells without doxycycline treatment. After treatment with doxycycline for $72 \mathrm{~h}$, both of p-ATM and p-BRCA1 in shHP1 $\gamma$-infected R-U937 cells and shHP1 $\gamma$-infected R-HL-60 cells was decreased (Figure 4). These results suggested that HP1 $\gamma$ contributed to maintain the constitutive activation of ATM/BRCA1 signaling in AZA-resistant cells.

\section{Treatment With Bromodomain Inhibitor Decreased HP1 $\gamma$ Protein}

Although our results indicated that HP1 $\gamma$ could be a novel molecular target in the treatment of myeloid neoplasms with AZA resistance, the small molecule targeting $\mathrm{HP} 1 \gamma$ is unfortunately unavailable. A relationship between sensitivity to bromodomain inhibitor and HP1 $\gamma$ expression was recently indicated (Knoechel et al., 2014), however, so we instead examined the effect of the bromodomain inhibitor I-BET151 on cell growth and HP1 $\gamma$ protein expression.

We treated the cells with $0.1,0.3,0.5,1,2,4$, and $8 \mu \mathrm{M}$ I-BET151 for 72 h. R-U937 cells (Figure 5A) and R-HL-60 cells (Figure 5B) showed higher sensitivity to I-BET151 in comparison with U937 cells and HL-60 cells, respectively. The IC50 values were $9.7 \mu \mathrm{M}$ in U937, $6.0 \mu \mathrm{M}$ in R-U937, 6.4 $\mu \mathrm{M}$ in HL-60 and $2.1 \mu \mathrm{M}$ in R-HL-60. Because these results indicated that 

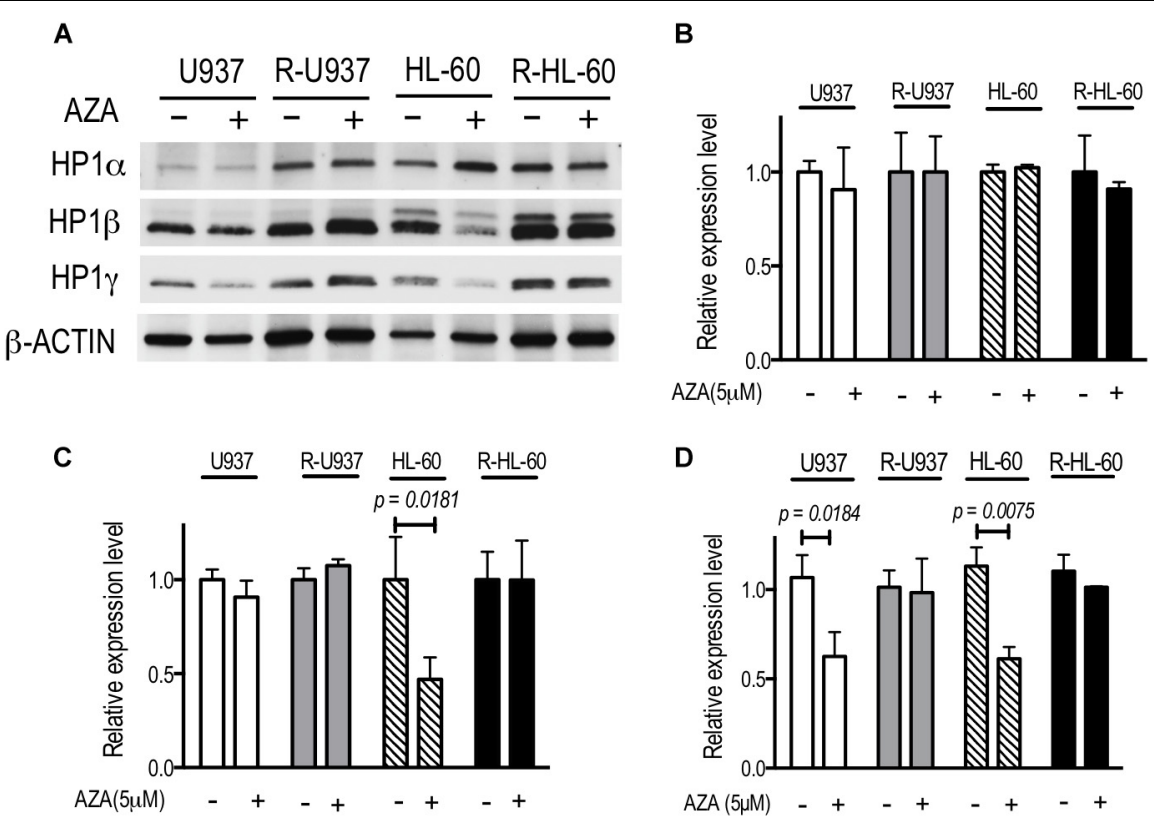

FIGURE 1 | (A) The protein expression of HP1 family members after AZA treatment at $5 \mu$ M for 72 h in U937, R-U937, HL-60, and R-HL-60 cells. Typical blots from a representative experiment are shown. The experiments were repeated three times. (B-D) Relative mRNA expression of HP1 $\alpha$ (B), $\beta$ (C), and $\gamma$ (D) after incubation with ( + ) or without (-) $5 \mu \mathrm{M}$ AZA for $72 \mathrm{~h}$ in U937, R-U937, HL-60, and R-HL-60 cells. The expression level was normalized with ACTB mRNA expression. The means $\pm \mathrm{SD}$ from three independent experiments are shown. $P$-values $<0.05$ were indicated.
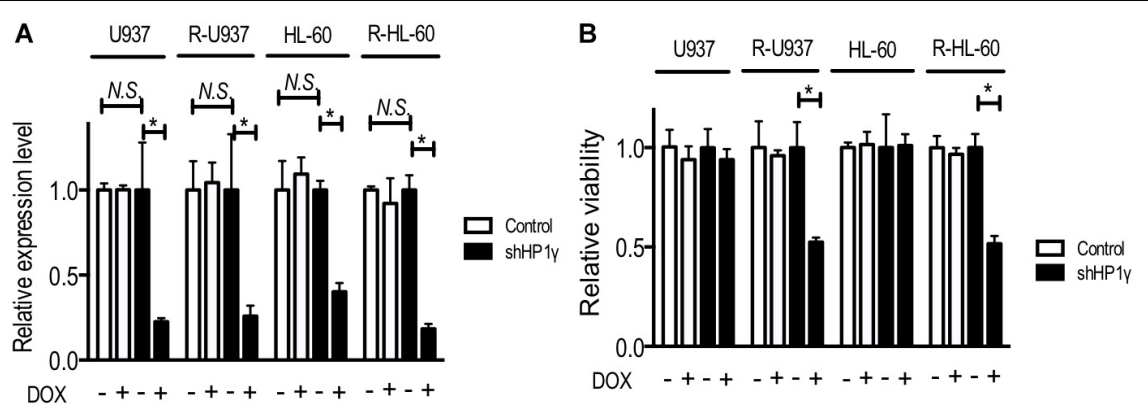

FIGURE 2 | (A) Relative mRNA expression of HP1 $\gamma$ after incubation with (+) or without (-) $1 \mu \mathrm{g} / \mathrm{ml}$ doxycycline (DOX) for $72 \mathrm{~h}$ in control plasmid-infected cells (white bars) and shHP1 $\gamma$-infected cells (black bars). The means \pm SD from three independent experiments are shown. ${ }^{*} p<0.0001$. (B) The relative viability of control plasmid-infected cells (white bars) and shHP1 $\gamma$-infected cells (black bars) after incubation with (+) or without (-) $1 \mu \mathrm{g} / \mathrm{ml} \mathrm{DOX}$ for $96 \mathrm{~h}$. The means $\pm \mathrm{SD}$ from three independent experiments are shown. ${ }^{*} p<0.0001$.

AZA-resistant cells were more sensitive to I-BET151 than their AZA-sensitive counterparts, we examined the effects of I-BET151 treatment on HP1 proteins (Figure 5C). The amount of HP1 $\alpha$ protein was not affected by treatment with I-BET151 in U937, R-U937, HL-60, and R-HL-60 cells. A reduction of HP1 $\beta$ protein was found only in HL-60 cells treated with $1 \mu \mathrm{M}$ I-BET151 for $48 \mathrm{~h}$, whereas other cell lines showed no such changes. Treatment with I-BET151 at $0.5 \mu \mathrm{M}$ for $48 \mathrm{~h}$ decreased HP1 $\gamma$ protein in U937 and R-U937 cells, and HP1 $\gamma$ protein in HL-60 and R-HL-60 cells was down-regulated after incubation with $1 \mu \mathrm{M}$ I-BET151 for $48 \mathrm{~h}$. In U937 and R-U937 cells, the decrease of HP1 $\gamma$ mRNA expression was detected after treatment with I-BET151 at $0.5 \mu \mathrm{M}$ for $48 \mathrm{~h}$ as well as in HL-60 and R-HL- 60 cells after treatment with I-BET151 at $1 \mu \mathrm{M}$ for $48 \mathrm{~h}$ (Figure 5D). The repression of mRNA expression was more notable in R-U937 and R-HL-60 cells in comparison with their AZA-sensitive counterparts, indicating an important role of bromodomain proteins in $\mathrm{HP} 1 \gamma$ expression in AZA-resistant cells.

\section{DISCUSSION}

In the epigenetic regulation of gene expression and chromatin, epigenetic writers, erasers, and readers play roles through direct and indirect interactions (Fuks et al., 2000, 2003; Vire et al., 2006; Meier and Brehm, 2014). Therefore, drugs targeting an epigenetic regulator can also affect other regulator proteins or epigenetic marks. Grövdal et al. (2014) showed that AZA treatment reduced 

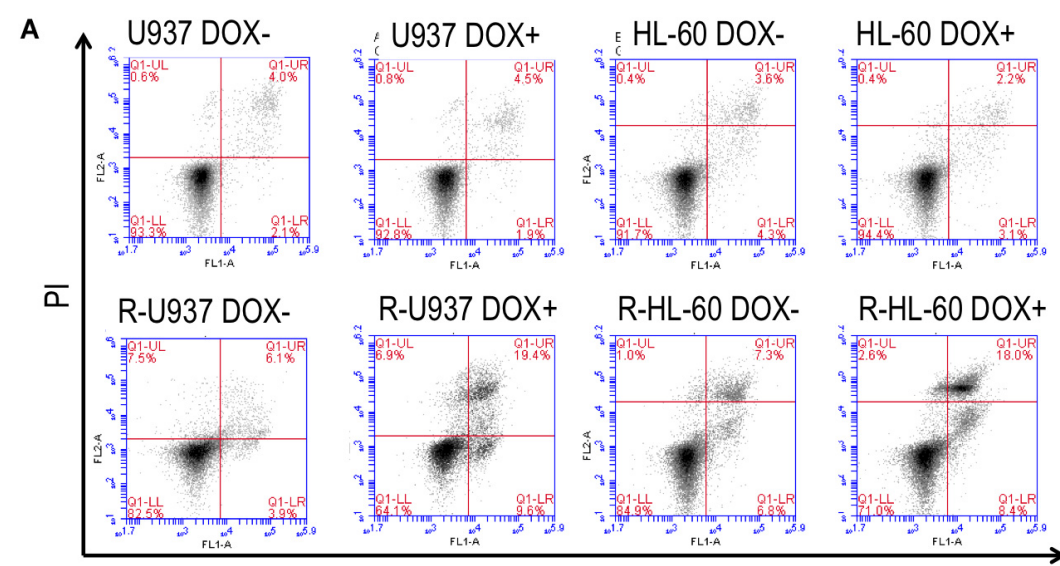

Annexin V

B

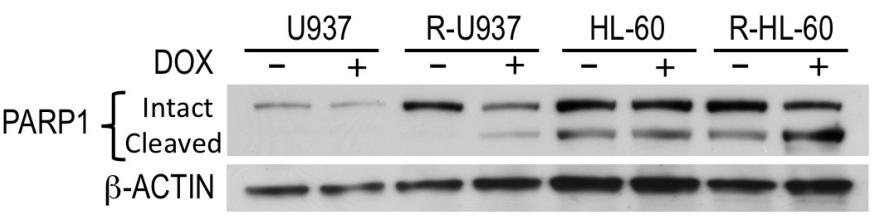

FIGURE 3 | (A) FACS analysis of annexin V/propidium iodide double staining in shHP1 $\gamma$-infected cells after incubation with (DOX+) or without (DOX-) $1 \mu \mathrm{g} / \mathrm{ml}$ doxycycline for $96 \mathrm{~h}$. Typical plots from a representative experiment were shown. The experiments were repeated three times. (B) The PARP1 cleavage profiles in shHP1 $\gamma$-infected cells after incubation with (DOX+) or without (DOX-) $1 \mu \mathrm{g} / \mathrm{ml}$ doxycycline for $96 \mathrm{~h}$.

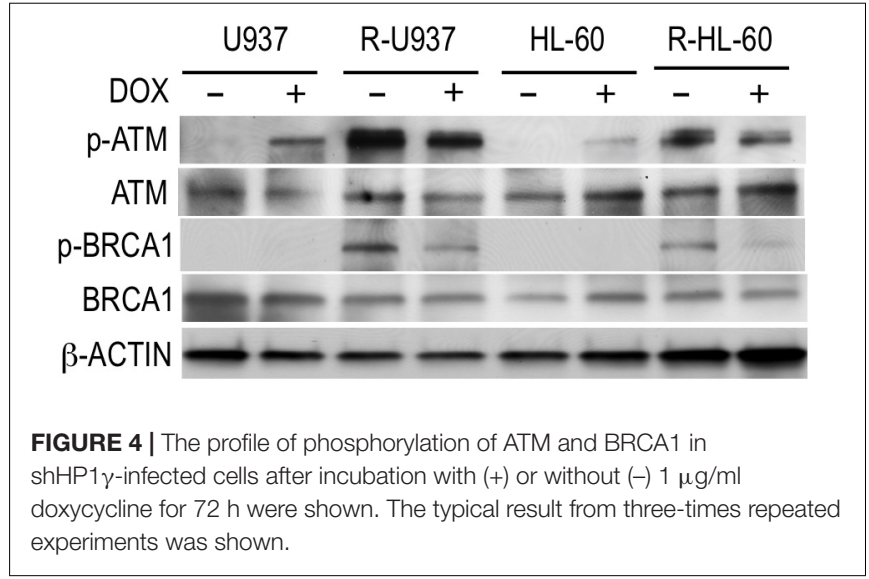

H3K27ac and H3K9ac and Tobiasson et al. (2017) reported increase of genes marked by $\mathrm{H} 3 \mathrm{~K} 9 \mathrm{me} 3$ after treatment with AZA in addition to genome-wide DNA demethylation in CD34+ progenitor cells obtained from patients with myelodysplastic syndromes. However, the effects of AZA on epigenetic reader were left unknown. In this study, we found that AZA treatment caused the decrease of HP1 $\gamma$ in AZA-sensitive cells but not in AZA-resistant cells. These observations are the first evidence that AZA can affect an epigenetic reader and indicate that the pharmacological activity of AZA might involve disruption of heterochromatin regulation via the methylated $\mathrm{H} 3 \mathrm{~K} 9 / \mathrm{HP} 1 \gamma$ axis.

Recently, Tobiasson et al. (2017) showed that treatment with $1 \mu \mathrm{M}$ AZA for $24 \mathrm{~h}$ increased the expression of genes marked by $\mathrm{H} 3 \mathrm{~K} 9 \mathrm{me} 3$ in $\mathrm{CD} 34+$ progenitor cells obtained from patients with myelodysplastic syndromes or myelodysplastic syndromerelated disease, but the changes in the $\mathrm{H} 3 \mathrm{~K} 9 \mathrm{me} 3$ profile could explain only a minor part of changes in the gene expression profile. Interestingly, they also showed that changes in the gene expression profile did not correlate with DNA demethylation. Such dissociation between gene expression and epigenetic marks could be explained by the dysregulation of epigenetic readers such as HP1 $\gamma$.

Members of the HP1 family, $\mathrm{HP} 1 \alpha, \beta$, and $\gamma$, recognize and bind to di- or tri-methylated $\mathrm{H} 3 \mathrm{~K} 9$, the epigenetic marks of heterochromatin, and function to compact heterochromatin (Zeng et al., 2010). However, some studies have shown that HP1 $\gamma$, unlike $\mathrm{HP} 1 \alpha$ and $\beta$, distributes to not only heterochromatin but also euchromatin (Lomberk et al., 2006; Zeng et al., 2010; Kwon and Workman, 2011). Lomberk et al. (2006) reported that phosphorylated HP1 $\gamma$ distributed on euchromatin interacts with $\mathrm{Ku} 70$, a regulatory protein in multiple nuclear processes. Another study by Vakoc et al. (2005) showed that HP1 $\gamma$ and H3K9me3 were associated with transcription elongation. Therefore, the down-regulation of HP1 $\gamma$ by AZA treatment indicates that some part of AZA activity might be caused by the disruption of multiple processes on euchromatin.

The knock-down experiment of HP1 $\gamma$ demonstrated that HP1 $\gamma$-dependent chromatin regulation, which could not be replaced by $\mathrm{HP} 1 \alpha$ and $\beta$, played an essential role in the survival of AZA-resistant cells. In this study, we showed that targeting HP1 $\gamma$ caused decrease of p-ATM and p-BRCA1 in AZA-resistant cells, suggesting that constitutive activation of ATM/BRCA1 signaling in AZA-resistant cells needs HP1 $\gamma$ function. Therefore, a possible 
A

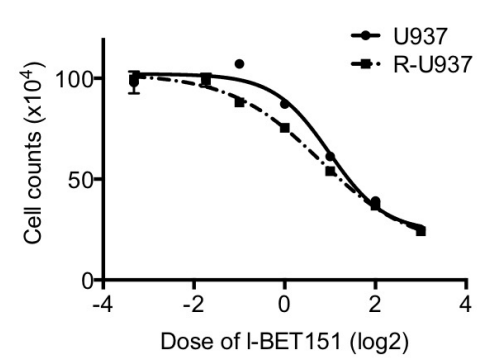

C

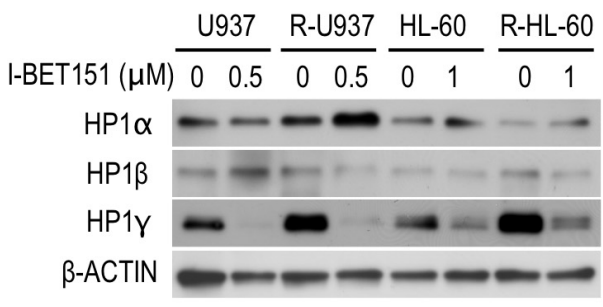

B

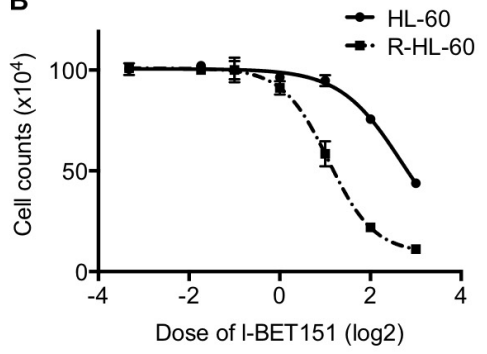

D

$\underline{\mathrm{U} 937} \quad \mathrm{R}-\mathrm{U937} \quad \underline{\mathrm{HL}-60} \underline{\mathrm{R}-\mathrm{HL}-60}$

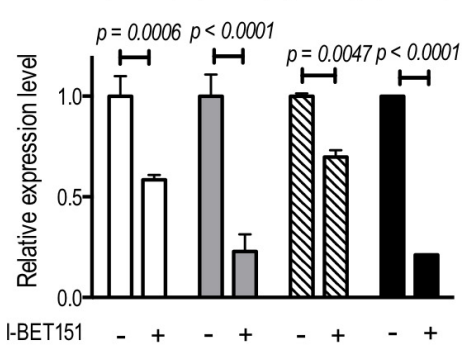

FIGURE 5 | The drug response curve using the cell counts of (A) U937 (solid line) and R-U937 cells (dotted line) and (B) HL-60 (solid line) and R-HL-60 cells (dotted line) after incubation with $0.1,0.3,0.5,1,2,4$, and $8 \mu \mathrm{M} \mathrm{I-BET151}$ for $72 \mathrm{~h}$. For each cell set, the mean $\pm \mathrm{SD}$ from three independent experiments are shown. (C) Protein expression levels of HP1 $\alpha, \beta$, and $\gamma$ in U937, R-U937, HL-60, and R-HL-60 cells after incubation with I-BET151 at indicated dose for 48 h. Typical blots from a representative experiment are shown. The experiments were repeated three times. (D) Relative mRNA expression of HP1 $\gamma$ after incubation with (+) or without (-) I-BET151 for $48 \mathrm{~h}$. The concentration of I-BET151 was $0.5 \mu \mathrm{M}$ for U937 and R-U937 cells and $1 \mu \mathrm{M}$ for HL-60 and R-HL-60 cells. The mean \pm SD from three independent experiments are shown. $P$-values $<0.05$ were indicated.

explanation is that a decrease of HP1 $\gamma$ caused growth inhibition and apoptosis via down-regulation of the constitutively activated DNA damage repair response in AZA-resistant cells. On the other hand, because HP1 $\gamma$ activity is involved in multiple processes on euchromatin, another possibility is that the disruption of some euchromatin process caused growth inhibition and apoptosis. In AZA-sensitive cells, the phosphorylation of BRCA1, but not that of ATM, was inhibited by HP1 $\gamma$ targeting. It is reported that the recruitment of ATM to the DNA damage site requires HP1 family members (Goodarzi et al., 2008). Wu et al. (2015) demonstrated that BRCA1 retention at the DNA damage site depends on interaction of BARD1 and HP1 $\gamma$. Our results in AZA-sensitive cells, which indicated the need of HP1 $\gamma$ to activate BRCA1 by ATM, well agree with these preceding studies. HP1 $\gamma$ targeting in AZA-sensitive cells might caused DNA damage and inhibited BRCA1 activation, while other DNA repair signaling such as PARP signaling might prevent growth inhibition and apoptosis in AZA-sensitive cells.

In spite of recent pharmaceutical developments focusing on the targeting of epigenetic readers (Dawson et al., 2012; Greschik et al., 2017; Zaware and Zhou, 2017), the small molecule targeting HP1 proteins is still unavailable. We found that I-BET151 downregulated $\mathrm{HP} 1 \gamma$ at transcriptional level in both AZA-resistant and AZA-sensitive cells, indicating that I-BET151 can work as an inhibitor of HP1 1 . Because the decrease of HP1 $1 \gamma$ showed a growth inhibitory effect specifically in AZA-resistant cells, the higher sensitivity to I-BET151 in AZA-resistant cells might involve the down-regulation of $\mathrm{HP} 1 \gamma$. A recent study reported that $\mathrm{T}$ cell acute lymphoblastic leukemia cells with resistance against $\gamma$-secretase inhibitor showed higher expression of HP1 $\gamma$ and higher sensitivity to another bromodomain inhibitor, JQ1, than those without $\gamma$-secretase inhibitor resistance (Knoechel et al., 2014). These observations appear to indicate that the sensitivity to bromodomain inhibitors is related to expression of HP1 $\gamma$ or dependence on HP1 $\gamma$. Further research into the relationship between bromodomain family euchromatin regulators and $\mathrm{HP} 1 \gamma$ is thus warranted.

Our approach had several limitations. Although the present results demonstrated that $\mathrm{HP} 1 \gamma$-specific function(s) contributed to the survival of AZA-resistant cells, it is still unclear which function(s) played the key role in their survival. The relationship between dependence on HP1 $\gamma$ and sensitivity to BET inhibition should be confirmed in leukemia cells obtained from patients with AZA resistance.

Although the involvement of many cellular processes in AZA activity have been studied, its effects on epigenetic reader proteins have never been examined. In this study, we demonstrated that AZA affects epigenetic reader proteins, such as HP1 $\gamma$. Our findings should help to improve our understanding of the mechanism(s) underlying AZA activity. One key finding is the importance of chromatin regulation by $\mathrm{HP} 1 \gamma$ in AZA-resistant cells. The agent targeting HP1 $\gamma$ still needs to be identified, but this study revealed the possibility that I-BET151 can work as a HP1 $\gamma$ inhibitor via down-regulation of HP1 1 . HP1 $1 \gamma$ targeting or bromodomain inhibition could be a novel strategy for the treatment of hematologic malignancies with AZA resistance. 


\section{AUTHOR CONTRIBUTIONS}

SI designated study, collected and analyzed data, and wrote the manuscript. TU and CK contributed data collections. TO provided the plasmids and advised for the experiments. $\mathrm{KO}$ and JO supervised the study. All authors reviewed the manuscript.

\section{REFERENCES}

Achille, N. J., Othus, M., Phelan, K., Zhang, S., Cooper, K., Godwin, J. E., et al. (2016). Association between early promoter-specific DNA methylation changes and outcome in older acute myeloid leukemia patients. Leuk. Res. 42, 68-74. doi: 10.1016/j.leukres.2016.01.004

Aimiuwu, J., Wang, H., Chen, P., Xie, Z., Wang, J., Liu, S., et al. (2012). RNA-dependent inhibition of ribonucleotide reductase is a major pathway for 5-azacytidine activity in acute myeloid leukemia. Blood 119, 5229-5238. doi: 10.1182/blood-2011-11-382226

Dawson, M. A., Kouzarides, T., and Huntly, B. J. (2012). Targeting epigenetic readers in cancer. N. Engl. J. Med. 367, 647-657. doi: 10.1056/NEJMra1112635

Fuks, F., Burgers, W. A., Brehm, A., Hughes-Davies, L., and Kouzarides, T. (2000). DNA methyltransferase Dnmt1 associates with histone deacetylase activity. Nat. Genet. 24, 88-91. doi: 10.1038/71750

Fuks, F., Hurd, P. J., Deplus, R., and Kouzarides, T. (2003). The DNA methyltransferases associate with $\mathrm{HP1}$ and the SUV39H1 histone methyltransferase. Nucleic Acids Res. 31, 2305-2312. doi: 10.1093/nar/gkg332

Goodarzi, A. A., Noon, A. T., Deckbar, D., Ziv, Y., Shiloh, Y., Lobrich, M., et al. (2008). ATM signaling facilitates repair of DNA double-strand breaks associated with heterochromatin. Mol. Cell. 31, 167-177. doi: 10.1016/j.molcel. 2008.05.017

Greschik, H., Schule, R., and Gunther, T. (2017). Selective targeting of epigenetic reader domains. Expert Opin. Drug Discov. 12, 449-463. doi: 10.1080/17460441. 2017.1303474

Grövdal, M., Karimi, M., Tobiasson, M., Reinius, L., Jansson, M., Ekwall, K., et al. (2014). Azacitidine induces profound genome-wide hypomethylation in primary myelodysplastic bone marrow cultures but may also reduce histone acetylation. Leukemia 28, 411-413. doi: 10.1038/leu.2013.265

Imanishi, S., Umezu, T., Ohtsuki, K., Kobayashi, C., Ohyashiki, K., and Ohyashiki, J. H. (2014). Constitutive activation of the ATM/BRCA1 pathway prevents DNA damage-induced apoptosis in 5-azacytidine-resistant cell lines. Biochem. Pharmacol. 89, 361-369. doi: 10.1016/j.bcp.2014.03.008

Knoechel, B., Roderick, J. E., Williamson, K. E., Zhu, J., Lohr, J. G., Cotton, M. J., et al. (2014). An epigenetic mechanism of resistance to targeted therapy in $\mathrm{T}$ cell acute lymphoblastic leukemia. Nat. Genet. 46, 364-370. doi: 10.1038/ng. 2913

Komashko, V. M., and Farnham, P. J. (2010). 5-azacytidine treatment reorganizes genomic histone modification patterns. Epigenetics 5, 229-240. doi: 10.4161/epi. 5.3.11409

Kwon, S. H., and Workman, J. L. (2011). The changing faces of HP1: from heterochromatin formation and gene silencing to euchromatic gene expression: HP1 acts as a positive regulator of transcription. Bioessays 33, 280-289. doi: 10.1002/bies.201000138

Lomberk, G., Bensi, D., Fernandez-Zapico, M. E., and Urrutia, R. (2006). Evidence for the existence of an HP1-mediated subcode within the histone code. Nat. Cell Biol. 8, 407-415. doi: 10.1038/ncb1383

Lund, K., Cole, J. J., Vanderkraats, N. D., Mcbryan, T., Pchelintsev, N. A., Clark, W., et al. (2014). DNMT inhibitors reverse a specific signature of aberrant promoter DNA methylation and associated gene silencing in AML. Genome Biol. 15:406. doi: 10.1186/s13059-014-0406-2

Meier, K., and Brehm, A. (2014). Chromatin regulation: how complex does it get? Epigenetics 9, 1485-1495. doi: 10.4161/15592294.2014.971580

Monika Belickova, M., Merkerova, M. D., Votavova, H., Valka, J., Vesela, J., Pejsova, B., et al. (2016). Up-regulation of ribosomal genes is associated with a poor response to azacitidine in myelodysplasia and related neoplasms. Int. J. Hematol. 104, 566-573. doi: 10.1007/s12185-016-2058-3

Moss, T. (2011). DNA methyltransferase inhibition may limit cancer cell growth by disrupting ribosome biogenesis. Epigenetics 6, 128-133. doi: 10.4161/epi.6.2. 13625

\section{FUNDING}

This work was supported by the Private University Strategic Research Based Support Project (Grant No. S1311016) to JO from MEXT, Tokyo, Japan, and a grant for medical research from the Takeda Science Foundation, Osaka, Japan, to SI.

Ohyashiki, J. H., Hisatomi, H., Nagao, K., Honda, S., Takaku, T., Zhang, Y., et al. (2005). Quantitative relationship between functionally active telomerase and major telomerase components (hTERT and hTR) in acute leukaemia cells. $\mathrm{Br}$. J. Cancer 92, 1942-1947. doi: 10.1038/sj.bjc.6602546

Saunthararajah, Y. (2013). Key clinical observations after 5-azacytidine and decitabine treatment of myelodysplastic syndromes suggest practical solutions for better outcomes. Hematology Am. Soc. Hematol. Educ. Program 2013, 511-521. doi: 10.1182/asheducation-2013.1.511

Stresemann, C., and Lyko, F. (2008). Modes of action of the DNA methyltransferase inhibitors azacytidine and decitabine. Int. J. Cancer 123, 8-13. doi: 10.1002/ijc. 23607

Tobiasson, M., Abdulkadir, H., Lennartsson, A., Katayama, S., Marabita, F., De Paepe, A., et al. (2017). Comprehensive mapping of the effects of azacitidine on DNA methylation, repressive/permissive histone marks and gene expression in primary cells from patients with MDS and MDS-related disease. Oncotarget 8, 28812-28825. doi: 10.18632/oncotarget.15807

Tsujioka, T., Yokoi, A., Itano, Y., Takahashi, K., Ouchida, M., Okamoto, S., et al. (2015). Five-aza-2' -deoxycytidine-induced hypomethylation of cholesterol 25hydroxylase gene is responsible for cell death of myelodysplasia/leukemia cells. Sci. Rep. 5:16709. doi: 10.1038/srep16709

Umezu, T., Ohyashiki, K., Kuroda, M., and Ohyashiki, J. H. (2013). Leukemia cell to endothelial cell communication via exosomal miRNAs. Oncogene 32, 2747-2755. doi: 10.1038/onc.2012.295

Vakoc, C. R., Mandat, S. A., Olenchock, B. A., and Blobel, G. A. (2005). Histone H3 lysine 9 methylation and HP1gamma are associated with transcription elongation through mammalian chromatin. Mol. Cell 19, 381-391. doi: 10.1016/ j.molcel.2005.06.011

Valencia, A., Masala, E., Rossi, A., Martino, A., Sanna, A., Buchi, F., et al. (2014). Expression of nucleoside-metabolizing enzymes in myelodysplastic syndromes and modulation of response to azacitidine. Leukemia 28, 621-628. doi: 10.1038/ leu.2013.330

Vire, E., Brenner, C., Deplus, R., Blanchon, L., Fraga, M., Didelot, C., et al. (2006). The Polycomb group protein EZH2 directly controls DNA methylation. Nature 439, 871-874. doi: 10.1038/nature04431

Wu, W., Nishikawa, H., Fukuda, T., Vittal, V., Asano, M., Miyoshi, Y., et al. (2015). Interaction of BARD1 and HP1 is required for BRCA1 retention at sites of DNA damage. Cancer Res. 75, 1311-1321. doi: 10.1158/0008-5472. CAN-14-2796

Zaware, N., and Zhou, M. M. (2017). Chemical modulators for epigenome reader domains as emerging epigenetic therapies for cancer and inflammation. Curr. Opin. Chem. Biol. 39, 116-125. doi: 10.1016/j.cbpa.2017.06.012

Zeng, W., Ball, A. R. Jr., and Yokomori, K. (2010). HP1: heterochromatin binding proteins working the genome. Epigenetics 5, 287-292. doi: 10.4161/epi.5.4. 11683

Conflict of Interest Statement: KO received research support from Celegene KK, served as a consultant and advisor of Celegene KK, and received honoraria for lecture fees from Nippon Shinyaku.

The remaining authors declare that the research was conducted in the absence of any commercial or financial relationships that could be construed as a potential conflict of interest.

Copyright (c) 2018 Imanishi, Umezu, Kobayashi, Ohta, Ohyashiki and Ohyashiki. This is an open-access article distributed under the terms of the Creative Commons Attribution License (CC BY). The use, distribution or reproduction in other forums is permitted, provided the original author(s) and the copyright owner(s) are credited and that the original publication in this journal is cited, in accordance with accepted academic practice. No use, distribution or reproduction is permitted which does not comply with these terms. 